\title{
Children's acceptance of Pilot program of School meals in rural region of northern Croatia
}

\author{
Helena Tomic-Obrdalj, Davorka Gajari and Jasmina Ranilovic \\ PODRAVKA Food Industry Inc., Research and Development, Koprivnica, Croatia
}

\section{Abstract}

In the rural region of northern Croatian county Koprivnica Krizevci, new school meals program "Smart meals for smart kids" was implemented since the beginning of 2019. Analysis of nutritional quality of standard school meals served in 18 primary schools indicated high consumption of cereal products, meat, particularly meat products, pastry and sweets, and lacking of fruits, vegetables, fish and legumes. New menu was designed with the aim of improving nutritional quality of schools' meals through a new recipes based on gastronomy heritage, dietary habits and National guidelines, but also education and testing of children's acceptance. Initial step of the project was to test new menu through Pilot program in six schools during two weeks. Process was accompanied by educational activities through originally designed leaflet about healthy diet, new meals and nutritional benefits, and was distributed among all students. Children's acceptance of Pilot menu of 10 meals was analyzed on a 9-point pictured hedonic scale and qualitative discussion through focus groups. In every school questionnaire was distributed in $4^{\text {th }}$ (age 9 to 10) and $7^{\text {th }}$ grade (age 12 to 13). In total, 237 children filled out the questionnaire, 104 from $4^{\text {th }}$ grade and 133 from $7^{\text {th }}$ grade. In focus groups, 26 participated 14 students and 12 teachers. Results show that best accepted meals were Spaghetti Bolognese, Breaded fish with cabbage savoy and potatoes, and Chicken risotto. High acceptance of this meals can be explained by familiarity to the children, often being served at school, relatively simple dishes with lesser amount of known ingredients and adequate taste profile. Least preferred meals were Chickpea with barley and corn, and Gnocchi with turnip, these meals were in most schools served for the first time, ingredients were unfamiliar, and combination of tastes was not according to the children's preferences. Qualitative discussion revealed that children noticed the difference from standard meals, particularly increase in serving of fruits and different and new varieties of vegetables, and believe that new meals are healthier. They also liked the educational leaflet and think it is a good way of communicating about healthy diet. Teachers welcome the new menus, differences were noticeable in intensity of spiciness, new varieties of vegetables and legumes, and more served fruits. For a successful and sustainable Program follow-up, school meals should be adapted to children's dietary habits gradually with careful introduction of the new food and tastes, followed by continuous nutritional education.

\section{Conflict of Interest}

There is no conflict of interest. 